\title{
Strumming a Strongly Interacting Fermi Gas
}

\author{
Sound waves reveal the unique properties of the unitary Fermi gas, a model system for \\ describing certain superconductors and forms of nuclear matter.
}

\section{by Tilman Enss ${ }^{1}$}

$\mathrm{T}$ he way a material transmits sound waves can reveal a lot about its properties. For instance, the lower the compressibilty of a medium, the faster sound waves will pass through it. In addition, a material undergoing a phase transition may attenuate sound more strongly, providing a signature of the change. Taking advantage of sound's qualities as a material probe, two independent teams have used it to understand the properties of the so-called unitary Fermi gas [1,2]. This form of quantum matter contains very strongly interacting fermions, and it is a model system for describing some superconductors and neutron stars. In their experiments, the researchers created the gas from a cloud of ultracold lithium atoms and explored the damping rate for sound waves in two regimes, showing that this rate reaches a universal lower quantum limit. The new findings may help scientists understand transport mechanisms in strongly interacting fermions found in condensed-matter or nuclear physics.

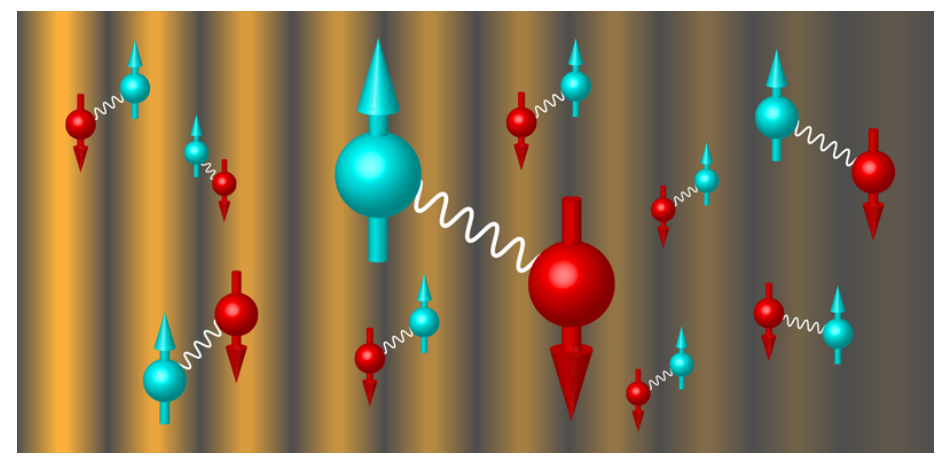

Figure 1: A sound wave passing through a strongly interacting gas of fermions known as the unitary Fermi gas. Two research groups have measured the damping rate of such waves to uncover the properties of the gas [1, 2]. (APS/Alan Stonebraker)

${ }^{1}$ Institute for Theoretical Physics, University of Heidelberg, Heidelberg, Germany
Quarks, electrons, neutrons, and many types of atoms are fermions, but they have vastly different characteristics. An electron, for instance, is a charged, fundamental particle, while an atom like lithium- 6 is a neutral, compound particle that's more than ten thousand times heavier. However, even seemingly different fermionic systems can exhibit surprisingly similar behavior if the interactions between the particles are sufficiently strong. That's why, for example, a tabletop experiment with a strongly interacting gas of atoms can be used to understand and predict certain properties of neutrons in a collapsed star.

The unitary Fermi gas is a special case of this general idea. If a dilute gas of neutral fermionic atoms is cooled to nanokelvin temperatures, then quantum effects will dominate, and the fermions will spread out because they cannot occupy the same quantum state. However, if a sufficiently strong attractive interaction is induced between the atoms, then they can bind into tight molecules that behave as bosons and bunch together. For a sweet spot value of the interaction, the atoms will behave neither purely as fermions nor bosons but will instead form a new state of quantum matter. This state is the unitary Fermi gas, and it has the strongest interaction between fermions allowed by quantum mechanics.

The unitary Fermi gas has very unusual transport properties. At low temperatures it can flow around obstacles with almost no friction [3], the hallmark of a perfect fluid. At lower temperatures still, it becomes a frictionless fluid, or superfluid [4]. The new studies examine what happens to sound waves as the unitary Fermi gas enters this superfluid phase. More specifically, they compare the observed behavior to that seen in the fermionic and bosonic versions of liquid helium, two traditional examples of a quantum fluid. Liquid helium-3 is fermionic, and when it nears its superfluid phase transition, sound waves passing through it experience a higher rate of damping. The opposite effect is seen in bosonic helium-4, in which the damping rate drops at the superfluid transition. Given that the particles in the unitary Fermi gas behave neither purely as fermions nor bosons, will the gas resemble either of the helium liquids, or will it behave as a new type of quantum fluid?

The two groups addressed this question by analyzing 
sound waves transmitted through unitary Fermi gases of ultracold lithium- 6 atoms in the vicinity of the superfluid transition temperature (Fig. 1). One group, led by Chris Vale of Swinburne University in Australia, studied sound waves with a relatively short wavelength. At these wavelengths, the superfluid phase is in the so-called collisionless regime, where sound waves are weakly damped by kicks from thermally excited particles [1]. The team focused two lasers with slightly different frequencies on a small region of a trapped atom cloud. This laser combination creates a density undulation in the gas, and by analyzing the momentum transferred to the gas for various frequency settings, the researchers could infer the damping rate of sound waves. They found that the damping rate drops suddenly when the gas transitions from the normal state to a superfluid. This feature agrees with the team's theory calculations [1], and its size is strikingly similar to the decrease observed in bosonic helium-4.

The other group, led by Martin Zwierlein at the Massachusetts Institute of Technology (MIT), Cambridge, focused on longer-wavelength sound waves [2]. In this "hydrodynamic" regime, the damping of sound waves occurs through a diffusive exchange of momentum and heat with the surrounding gas. The team used several overlapping laser beams to trap the gas in a rectangular "box", ensuring that the gas had a uniform density throughout the trap and that the properties of sound waves were the same everywhere. The researchers excited a sound wave that travels across the gas by effectively "tapping" one side of the box (see Making Waves in a "Glass" Full of Atoms.) They then imaged the atomic density to directly observe the wavelength and damping rate of the traveling wave. Cooling the gas through the superfluid phase transition, they found a gradual dropoff in the diffusive damping rate of sound waves. Eventually, the diffusion coefficient (which factors into the damping rate) approached a quantum limit of $\sim$ $\hbar / m$, where $\hbar$ is Planck's constant and $m$ is the lithium atom's mass.

Theoretical work also predicts a lower limit on the diffusion coefficient. The value of this limit is set by the Heisenberg uncertainty principle, which prevents diffusion from becoming arbitrarily slow [5]. The same universal value has been observed in strongly interacting bosonic helium-4. But unlike helium-4, the unitary Fermi gas in the hydrodynamic regime of the MIT experiments showed no sudden dropoff in damping at the superfluid phase transition. This finding suggests that the damping mechanism in the unitary Fermi gas may involve the breaking of fermion pairs, which occurs in helium- 3 but has no counterpart in bosonic helium-4.

Taken together, the two studies show that the unitary Fermi gas shares certain "universal" characteristics with helium -3 and with helium-4. But it is not completely like one or the other. Finding such universalities is exciting because it links cold atoms to other fields of physics, while understanding the subtle deviations from universality will reveal the unique attributes of the unitary Fermi gas. To this end, it's an achievement that these new experiments, as well as one reported last year [6], now allow sound waves to be measured in a region of homogeneous (uniform density) gas. The waves' behavior can thus be determined with high precision, allowing a more useful comparison with theory and opening a new chapter in our understanding of strongly interacting quantum fluids. For instance, measurements of the diffusion coefficients near the superfluid phase transition might soon detect predicted critical-scaling effects [5, 7]. Already, predicted quantum bounds related to spin diffusion [8] and to sound propagation in a 2D system [9] have been observed in experiments. These data will help to identify and understand common mechanisms for quantum limited, or "Planckian," transport [10], which has also been seen in high-temperature superconductors [11].

This research is published in Physical Review Letters and posted on the arXiv.

\section{REFERENCES}

[1] C. C. N. Kuhn et al., "High-frequency sound in a unitary Fermi gas," Phys. Rev. Lett. 124, 150401 (2020).

[2] P. B. Patel et al., "Universal sound diffusion in a strongly interacting Fermi gas," arXiv:1909.02555.

[3] T. Schäfer and D. Teaney, "Nearly perfect fluidity: From cold atomic gases to hot quark gluon plasmas," Rep. Prog. Phys. 72, 126001 (2009).

[4] L. A. Sidorenkov et al., "Second sound and the superfluid fraction in a Fermi gas with resonant interactions," Nature 498, 78 (2013).

[5] B. Frank et al., "Quantum critical thermal transport in the unitary Fermi gas," arXiv:2003.10338.

[6] L. Baird et al., "Measuring the hydrodynamic linear response of a unitary Fermi gas," Phys. Rev. Lett. 123, 160402 (2019).

[7] P. C. Hohenberg and B. I. Halperin, "Theory of dynamic critical phenomena," Rev. Mod. Phys. 49, 435 (1977).

[8] T. Enss and J. H. Thywissen, "Universal spin transport and quantum bounds for unitary fermions," Annu. Rev. Condens. Matter Phys. 10, 85 (2019).

[9] M. Bohlen et al., "Sound propagation and quantum limited damping in a two-dimensional Fermi gas," arXiv:2003.02713.

[10] A. Lucas, "Operator size at finite temperature and Planckian bounds on quantum dynamics," Phys. Rev. Lett. 122, 216601 (2019).

[11] B. Keimer et al., "From quantum matter to high-temperature superconductivity in copper oxides," Nature 518, 179 (2015).

10.1103/Physics. 13.53 